\title{
Mitigate risk of healthcare delivery system collapse: Some measures of Taiwan
}

\author{
Wen-Hui Su${ }^{1}$, Wei-Ling Huang ${ }^{2 *}$, Pei-Shan Shie ${ }^{3}$, Lin-Kun Wu ${ }^{4}$ and Shih-Huai Hsiao ${ }^{5,67 *}$ \\ ${ }^{1}$ Master of Science in Healthcare Administration; Director of Special Affairs Office, Administration Center, Kaohsiung Medical University Hospital, Kaohsiung \\ Medical University, Kaohsiung, Taiwan \\ ${ }^{2}$ Master of Business Administration; Head of Long-term care center, Kaohsiung Municipal Ta-Tung Hospital, Kaohsiung Medical University, Kaohsiung, Taiwan \\ ${ }^{3}$ Master of Science in Healthcare Administration; Head of Document Management Department, Administration Center, Kaohsiung Medical University Hospital, \\ Kaohsiung Medical University, Kaohsiung, Taiwan \\ ${ }^{4}$ RN, Master of Science; Deputy Director of Administration Center, Kaohsiung Medical University Hospital, Kaohsiung Medical University, Kaohsiung, Taiwan \\ ${ }^{5}$ Lecturer, Master of Public Health, Department of Public Health, Kaohsiung Medical University, Kaohsiung, Taiwan \\ ${ }^{6}$ Vice Superintendent, Kaohsiung Municipal Ta-Tung Hospital, Kaohsiung Medical University, Kaohsiung, Taiwan \\ ${ }^{7}$ Board Member, Taiwan College of Healthcare Executives, Taipei, Taiwan
}

Upon July 20, 2020, accumulated 14,281,172 people had confirmed SARS-CoV-2 infection and 602,068 people died of Coronavirus disease 2019 (COVID-19) in the world. It is a daunting challenge to cope with a surge of suspected infectious patients in the healthcare delivery systems.

Taiwan's achievement in thwarting a disease outbreak has been recognized globally. Some effective containment measures of the COVID-19 were adopted to prevent health care-associated infections (HAIs) at four Kaohsiung Medical University affiliated hospitals which provide over 3.5 million outpatient visits and 64 thousand admissions per year in the Central of Kaohsiung City with over 2.9 million citizens, we hope our parts of experience can be copies to Africa because the service of healthcare professionals has surely never been in greater need.

1. to complete of a TOCC history (travel, occupation, contact and cluster) sheet for every visitor before entering hospital [1].

2. to take patients' body temperature for a second time after acclimatizing to being indoors even though they pass infrared temperature detectors and forehead thermometers [2].

3. to enforce the reporting of Healthcare workers with unknown fever, acute respiratory illness and other symptoms.

4. to enforce restrictions on hospital visits and use on-site and web visitor real-name registration to record and trace all the patients' visitors.

5. to suspend cross-team nursing care included nursing shift hand-off occurs by team, nursing team members are not allowed to support direct care activities of a different team in the same ward, nursing team members are not be allowed to transfer patients to other wards or rooms, nursing team members must maintain social distancing during meetings, the number of nursing staff members in the locker room simultaneously is strictly limited, and team members must refrain from conversing during meals [3].

6. to set up outdoor dispensary counters for drug receiving without need to enter hospital should be one of the requisite measures for drug dispensing services [4].

7. to conduct ongoing surveillance to obtain early warning of potential contagion exposure.
8. to perform epidemiological investigation and outbreak management.

9. to installed air exhaust fans on the windows to change the pressure direction within the wards rapidly, a general inpatient ward area with 30 beds can easily, quickly and effectively be transformed into makeshift negative-pressure rooms within 24 hours. The best location for the fans was ninety centimetres from the floor, and ninety centimetres from the edge of bed whether the indoor air conditioners were on or off [5].

After the abovementioned containment COVID-19 measures were initiated, the medical quality and patient safety commissions at four hospitals also inspected those and ensured that all healthcare workers clearly understood and implemented the epidemic prevention measures required to be taken at different epidemic response levels. The end of COVID-19 global epidemic is not expected any time soon, although so far there have only been 55 community-acquired infection cases in Taiwan. Fortunately, there are no health care-associated infections at our four hospitals.

\section{Acknowledgement}

We thank all members of the COVID-19 Response Team of Kaohsiung Medical University Hospital for implementing the listed measures of containing COVID-19 and sharing their valuable experience of fighting COVID-19. None of the staff members mentioned from those departments received any compensation for their contributions. We also express gratitude to all trustees of the Board of Kaohsiung Medical University Trustees for supporting us with a lot of resource to establish a healthy and safe workplace for healthcare workers.

${ }^{\star}$ Correspondence to: Wei-Ling Huang, Head of Long-term care center, Kaohsiung Municipal Ta-Tung Hospital, Kaohsiung Medical University, Kaohsiung, Taiwan, E-mail: 1040166@kmuh.org.tw; helenhelen1531@hotmail. com

Key words: health care-associated infections, covid-19, healthcare workers, team nursing, nosocomial transmission, sars-cov-2

Received: July 15, 2020; Accepted: July 17, 2020; Published: July 31, 2020 


\section{Ethics approval}

As this is a descriptive study based on data collected by the hospital's Department of ICD, OOSH, and Human Resource Department as part of the outbreak management, ethics approval is not required under our healthcare system's Institutional Review Board guidelines.

\section{References}

1. Yang C-J, Chen T-C, Chen Y-H (2020) The preventive strategies of community hospital in the battle of fighting pandemic COVID-19 in Taiwan. J Microbiol Immunol Infect 53: 381. [Crossref]
2. Hsiao S-H, Chen T-C, Chien H-C, Yang C-J, Chen Y-H (2020) Measurement of body temperature to prevent pandemic COVID-19 in hospitals in Taiwan: repeated measurement is necessary. J Hosp Infect 105: 360-1. [Crossref]

3. Chen L-C, Wu L-K, Chen M-H (2020) Suspending Cross-Team Nursing Care Is Necessary to Prevent Health Care-associated Infection (HAI) during COVID-19. Kaohsiung Journal of Medical Sciences. (in-press)

4. Hsiao S-H, Chang H-M, Kang Y-T, Chen Y-H (2020) Mitigate Risks of Medication Interruption Due to COVID-19 for Elderly With Chronic Diseases. Kaohsiung J Med Sci. 10.1002/kjm2.12263[Crossref]

5. Huang W-N, Zhuang M-S, Cheng T-J, Hsiao S-H (2020) Simplest Way to Establish COVID-19 Quarantine Observation Wards within 24 Hours. Asia-Pacific Journal of Public Health. (in press).

Copyright: $\odot 2020$ Wen-Hui Su. This is an open-access article distributed under the terms of the Creative Commons Attribution License, which permits unrestricted use, distribution, and reproduction in any medium, provided the original author and source are credited. 